\title{
The Potential to Infer the Historical Pattern of Cultural
}

Macroevolution

3

\begin{abstract}
Dieter Lukas ${ }^{1 *}$, Mary Towner ${ }^{2}$, Monique Borgerhoff Mulder ${ }^{1,3}$
1) Department of Human Behavior, Ecology \& Culture, Max Planck Institute for Evolutionary Anthropology, Deutscher Platz 6, 04103 Leipzig, Germany; * author for correspondence (dieter_lukas@eva.mpg.de). Department of Integrative Biology, Oklahoma State University, Stillwater, OK 74078, USA Department of Human Behavior, Ecology \& Culture, Max Planck Institute for Evolutionary Anthropology Deutscher Platz 6, 04103 Leipzig, Germany; Department of Anthropology, University of California Davis, Davis, CA 95616, USA
\end{abstract}

\section{Summary}

Phylogenetic analyses increasingly take centre-stage in our understanding of the processes shaping patterns of cultural diversity and cultural evolution over time. Just as biologists explain the origins and maintenance of trait differences among organisms using phylogenetic methods, so anthropologists studying cultural macroevolutionary processes use phylogenetic methods to uncover the history of human populations and the dynamics of culturally transmitted traits. In this paper we revisit concerns with the validity of these methods. Specifically, we use simulations to reveal how properties of the sample (size, missing data), properties of the tree (shape), and properties of the traits (rate of change, number of variants, transmission mode) might influence the inferences that can be drawn about trait distributions across a given phylogeny and the power to discern alternative histories. Our approach shows that in two example datasets specific combinations of properties of the sample, of the tree, and of the trait can lead to potentially high rates of Type I and Type II errors. We offer this simulation tool to help assess the potential impact of this list of persistent perils in future cultural macroevolutionary work. 


\section{Introduction}

30 Human societies exhibit a diversity of cultural practices around the world [e.g., 1]. The

31 field of cultural macroevolution aims to identify the origins of this diversity and the factors

32 shaping the distribution of cultural variation across societies [2]. It is now almost obligatory

33 for studies investigating cross-cultural variation to link cultural practices to phylogenies

34 derived from linguistic, morphological, or genetic data [3-5]. Phylogenetic information is

35 often included in comparative studies, when testing for associations in the distribution of

36 cultural variation, to account for the potential dependencies that arise among traits from

37 a shared history. In addition, to fully understand the history of cultural traits and to

38 determine whether any association among cultural traits does reflect a causal

39 relationship, phylogenetic reconstructions of trait evolution trace changes in cultural

40 practices across a tree reflecting ancestral relationships among the societies.

41 Phylogenetic reconstructions of trait evolution offer the potential to infer what cultural

42 variants might have been present in the past, how variants change from one state to

43 another, and what socio-ecological conditions might have influenced such trajectories of

44 change [6]. As such they allow us to test a wide range of hypotheses for the patterning of

45 human cultural variability, bringing precision to the pursuits of early anthropologists, such

46 as Boas' [7] interest in separating the roles of culture, environment and biology, or

47 Murdock's [8] proposal that changes in residence rules precede change in other social 48 structures.

50 Phylogenetic reconstruction of trait evolution relies on two steps: the first is to infer the

51 likely historical relationships among populations, and the second is to determine whether 
52 changes in a cultural trait relate to the patterns of historical splits among these

53 populations. Here, we focus on the second part: we assume that a tree is available, and

54 that we want to understand where and when on the tree changes in the cultural traits

55 occurred. In most instances, we do not have information from the past to guide our

56 inferences of the history of a trait. In effect, phylogenetic reconstructions of trait evolution

57 are not opening a window onto the past, but painting a picture about the past based

58 primarily on information from the present.

60 The accuracy of this picture depends on how well we address at least four challenges.

61 The first is to assess how much the past is likely to resemble the present. If traits change

62 rapidly, we cannot say with certainty which of the variants at the tips of the phylogeny

63 might have been present at a particular point in the past, which in our painting metaphor

64 would be akin to the mix of colours from neighbouring societies leading to brown and

65 blurry internal nodes. Second, the accuracy of the picture is fundamentally affected by

66 the assumptions we use to link the current data to patterns in the past, and whether we

67 use appropriate models of rate and directionality in the transmission of cultural variants.

68 In terms of the metaphor, are we even using the right brush for this type of paint to capture

69 our depiction of the past? These first two challenges question whether a phylogenetic

70 method is appropriate to make inferences about the past for the particular trait. Third, the

71 accuracy will depend on how complete our present information is, on whether we have

72 an adequate sampling of cultural practices to make proper inferences about the past. In

73 terms of the metaphor, do we have the full range of colours in our palette or are some of

74 them missing, and if so why? Fourth, and related, what if there are traits that predominate 
75 in the present that did not exist in the past? We may be working with an entirely

76 inappropriate paint box. The last two challenges relate to the sample available to answer

77 a specific question about the past.

79 Many studies have investigated the strength of cultural ancestry of various traits by 80 mapping them onto an independent language phylogeny, and then directly evaluate their

81 fit with population history in order to detect phylogenetic signal. For example, Moylan et

82 al [9] examined the distribution of 55 East African cultural traits across a linguistic

83 phylogeny and found that only 18 showed a clear phylogenetic branching pattern.

84 Subsequent studies across multiple cultural domains report widely varying phylogenetic

85 signals for both material [10] and social organizational traits [11], signals that can also

86 vary by scale and the prevalence of cultural boundaries [12, 13]. Even across non-

87 humans, behavioural traits often show very low phylogenetic signal compared to

88 morphological and physiological traits [as in non-human primates, 14] and other animals

89 [15]. This widely varying strength of cultural ancestry likely reflects an interplay of the

90 factors listed above, raising multiple challenges for phylogenetic analyses of cultural

91 traits. These include (a) the extent to which the history of a trait can be reliably

92 reconstructed using population history (as captured in a phylogeny), (b) whether traits

93 change primarily at evolutionary time scales, and (c) whether as investigators we have

94 the appropriate data and (d) samples from which to infer past trait states.

95

96 We first examine these four challenges (Section 2) and next present simulations (Section

97 3) to illustrate some implications of these challenges for inferences in studies of trait 
98 evolution. We end (Section 4) with a discussion of how and why advances in addressing

99 these challenges can make phylogenetic approaches a powerful tool for understanding

100 human cultural diversity. The challenges we review are not fundamental flaws that prohibit

101 cultural phylogenetic approaches, but highlight our need to know the extent to which they

102 affect inferences drawn from phylogenetic analyses. Accordingly, we offer a checklist and

103 our simulation as part of a potential workflow assessing the challenges researchers using

104 phylogenetic methods face, in the spirit of "caveat emptor."

105

\section{Review of the challenges}

107 (a) To what extent can the history of a trait be reflected by a phylogeny?

108 (i) Are cultural traits inherited together or do they have independent histories?

109 Just as biologists recognize that every gene has its own history, so social scientists

110 appreciate this could also be true for cultural traits [e.g., 16, 17]. Boyd et al [18] evaluate

111 how human societies differ with respect to how integrated are their cultural traits. At one

112 end of the continuum, societies are seen as consisting of a tightly-knit set of cultural traits,

113 while at the other end, of largely ephemeral traits with only low coherence. Generally, the

114 empirical evidence points to the middle of the continuum, with societies containing a

115 vertically transmitted core of integrated cultural traits in addition to a hugely varying

116 proportion of more peripheral traits, some horizontally borrowed from other populations

117 and some independently invented. Bayesian phylogenetic analyses can be used to

118 identify incongruent cultural histories, by enabling researchers to classify traits as core or

119 peripheral and then test whether allowing rates of change between partitions provides a

120 better fit with the data than assuming equal rates of change [19]. While this approach can 
121 show how different traits are likely to have had different histories, it cannot reveal the

122 particular history of the individual traits. This leaves unresolved a determination of the

123 extent to which an independently-derived phylogeny can capture the distinct histories of

124 different culturally transmitted traits.

126 (ii) Can we determine how much deviations from exclusive vertical transmission of traits 127 will affect our ability to infer internal nodes?

128 Various approaches have been suggested to identify the role of horizontal transmission, 129 some retaining and others abandoning tree-based approaches. For example, biologists 130 explicitly model the possibility that not all genes within an individual will necessarily share 131 the same branching phylogeny [reconciliation analysis, 20] [incomplete lineage sorting, 132 21], a method that can be applied to human cultural traits $[19,22,23]$. Cultural 133 evolutionists turn to network analysis [24] or popularity spectra [25] to detect horizontal 134 transmission, shown in each of these cited studies to be particularly predominant in 135 oceanic environments. Other comparative social and evolutionary scientists turn to 136 various matrix frameworks, employing multiple regression, Mantel tests and autologistic 137 regression models to detect shared ancestry and/or cultural diffusion in their data [e.g., $13826,27-30]$. While these latter alternatives can indicate the relative contributions of vertical 139 versus horizontal transmission, there is generally no direct way to link them back to inform 140 inferences of internal nodes.

142 Simulation studies are particularly helpful in examining the sensitivity of inferences about 143 evolutionary processes to horizontal transmission. Nunn et al [31] studied character 
144 evolution in a spatial framework, and showed that horizontal transmission can in some

145 cases produce misleading inferences about evolutionary processes. Others show that

146 identifying patterns of trait evolution, and trait values at internal nodes, depends not just

147 of rates of borrowing but on whether traits are borrowed as packets or singly [32] [but see

148 discussion in 33], or advise on focusing on less "unrealistic" rates of borrowing [34]. But,

149 and this is the point, we rarely have direct windows onto the past, so speculations over

150 whether traits are borrowed as packages or singly, and at what rate, are questionable

$151[18,19]$. Decisions about what to consider a tolerable rate of borrowing or how to include

152 borrowing explicitly in any analysis depends on what we know empirically, on the nature

153 of the trait, and on whether the borrowing is global or local [as discussed in $24,25,31$, 154 32, 33].

156 (b) Does the trait change over evolutionary or shorter time scales?

157 (i) Can we identify the rate at which societies change their cultural traits?

158 If cultural traits are highly facultative, and change over very short periods of time, they 159 may not be amenable to phylogenetic reconstructions of their evolutionary history 160 because there is simply too much variation at the tips of the trees. The signature of history 161 might be weak compared to the strength of current selection pressures [35, 36]. Indeed, 162 simulations show that high rates of evolutionary change have a strong depressive impact 163 on measures of phylogenetic signal when determined through the fit of such traits onto 164 an independently determined tree [the "true" tree, see 33]. Estimating highly

165 facultative/flexible traits on an independently-derived tree risks obscuring the possibility 166 that there were in fact multiple undetectable changes on each branch. A phylogenetic 
167 approach cannot accurately reconstruct internal nodes if these do not retain a signal

168 about the past [37] and might instead present a regression to the mean with larger

169 changes earlier in the tree where deeper branches merge.

171 The problem of highly facultative traits is well-illustrated in a recent innovative study

172 linking archaeological and contemporary ethnographic data to examine the association

173 between dwelling size and post-marital residence [38]. By plotting dwelling size and

174 residence rules onto a time-calibrated global super-tree of human populations the authors

175 show that changes in house size precede changes in residence patterns. While there are

176 possible explanations for these findings, it is hard to see intuitively how dwelling size

177 (which might entail simply adding an extra room) is less mutable over evolutionary time

178 than post-marital residence norms (which are known to be vary with other traits such as

179 forms of property transmission, lineality, and warfare). Dwelling size is by no means a

180 uniquely facultative trait; polygyny, for example, also appears to be highly volatile [36,

181 39], raising the question of whether it is ever reasonable to put highly facultative traits on

182 a phylogeny built on language evolution $[35,40,41]$ ?

184 Identifying rates of change and flexibility is complicated. Short of a time machine, direct 185 evidence from history, archaeology or paleoanthropology is obviously the gold standard 186 [41]. The preferred method for inferring past changes is to identify an independent source 187 revealing the historical relationship among populations (step 1, as noted above), typically 188 a linguistic or genetic tree with known branch lengths. Investigators then use the observed 189 (tip) value of cultural traits to estimate (with probabilities) past values of the cultural trait 
190 at its internal nodes, and where possible triangulating with independent sources of data,

191 such as archaeological data [e.g., 42] or other well-known (and typically more recent)

192 cultural sequences [such as the technological changes in brasswind cornets, 43].

193 However, for many behavioural and cultural traits such historical data are unavailable.

195 A different approach entails making predictions, both from intuition and empirical 196 patterning, about the likely conservatism or volatility of traits in different domains: family 197 and kin-based traits, for example, have long been held by anthropologists to change 198 slowly [e.g., 44], or at least more slowly than traits directly linked to the environment [45,

199 46], but other patterns can emerge. Evidence from the Austronesian language family 200 shows phylogenetic and geographic (or both) patterning of some of the social/kinship 201 traits studied, but no clear model for just under half the 78 traits studied [11]. Similarly, 202 material and technological traits can show widely varying phylogenetic signal $[26,46,47]$. 203

204 (ii) Does the granularity at which cultural traits are defined affect the inferred rate of 205 change?

206 Any measured rate of trait replacement depends on the granularity of trait measurement 207 and categorization. This is particularly likely to be a problem for cultural traits, which are 208 often reported as categorical states (such as bridewealth, dowry, brideservice, and no 209 payments), states that are in many instances further combined into binary categories to 210 retain statistical power for analysis [48]. Transition rates may reflect such granularity, for

211 example a shift from matrilocality to non-matrilocality could take longer than a shift to 212 multilocality, which encompasses matrilocality (see Section 2biii). The difficulty of inferring 
213 transition rates is further compounded if the rate differs in different parts of the tree [49]

214 or if it is higher for some transitions than for others [50]. Finally studies of contemporary

215 cultural transitions, such as how bridewealth payments shift into effective dowry [51, 52]

216 or how matrifocal institutions emerge and wither [53,54], can throw light on the stability

217 and/or changeability of these specific traits, but generalization to other contexts remains

218 problematic.

220 In short, we still grapple with deriving a priori hypotheses for which cultural domains are

221 most conserved as well as with developing sound methods for determining how rapidly

222 they change. While the ancestral relationships among variants of cultural traits can be

223 represented as trees using "the most parsimonious cladogram" [55, page 175], the

224 calculation of indices to represent historical changes from such cladograms is problematic

225 [33, Section 4d]. It is worth stressing that the vast majority of traits show little or no

226 patterning with demographic and/or geographic indices that are supposed to reflect

227 population histories [e.g., 11, 36, 56]. This all points to the importance of facultative 228 adaptation across different domains, as envisaged by Rogers and Cashdan [35]. We

229 should then, as previously emphasized [e.g., 19, 57], acknowledge huge variability in the

230 rates at which traits change across domains, world regions, historical periods, and 231 geographic scales before deciding whether or not a phylogenetic approach is appropriate 232 for our particular trait of interest.

233

234 (iii) Are the traits of interest measurable at a scale whereby the question can be 235 satisfactorily answered? 
236 Closely related to the argument above, the grain at which traits are measured may

237 preclude, or even distort, understanding of evolutionary dynamics [58]. Comparisons

238 across large samples of diverse societies raise challenges for identifying traits that can

239 be both measured in all societies and considered homologous. A trait that appears similar

240 in different societies because it appears in the same context (e.g., ensuring food security

241 in agriculturalists and foragers) might represent independent inventions or borrowing [18].

243 The granularity at which traits are measured is likely to affect our ability to detect 244 sequences of cultural transitions, whether $A$ is a necessary precondition to $B$ or vice 245 versa. For example, consider "social complexity", a central trait in the debate over whether 246 moral gods precede or postdate the emergence of complex societies [59,60]. In an 247 examination of the robusticity of the measure of social complexity Miranda and Freedman 248 [61] show how two societies with radically different social organizations can look the same 249 in terms of a unidimensional measure of social complexity. By exploring beyond the first 250 principal component of a society's social complexity ranking [see also 62], the authors 251 develop a scale that could add considerable nuance to the conclusion (made from 252 transition analyses) that societal complexity causally preceded moralizing gods. The fact 253 that often only coarse codings are available in no way invalidates phylogenetic (or any 254 other comparative) analyses, but clearly impedes the strength of precise inference, 255 particularly with respect to sequences [48]. Furthermore, it bears stressing that breaking 256 down traits into multiple states will likely make it harder to identify phylogenetic signal, if 257 finer grain traits are more likely to change (as discussed in Section 2bii). 
259 More generally, for each of these data-related problems discussed in this section, starting

260 from a clear theoretical framework that outlines the specific predicted relationships among

261 well-defined variables can help to decide whether the empirical data available are

262 adequate to the question at hand. For example, to understand the evolution of a concept

263 such as "social complexity," now that social scientists recognize its general patterning we

264 might learn more from examining the distribution of specific social traits associated with

265 complexity [as in 63 , for non-humans] rather than continue to employ coarse

266 unidimensional variables. Where binarization is unavoidable, it should be based on clear

267 theoretical justification.

269 (c) Are there appropriate independently-derived population trees available for 270 phylogenetic analysis?

271 (i) Is there an appropriate tree to study the trait of interest at the scale of interest?

272 Assuming use of a phylogenetic tree is deemed appropriate, the next question is whether

273 the tree available well reflects the demographic history of the culture-bearing population.

274 With the use of genetic or, more commonly, linguistic trees, an affirmative answer seems

275 reasonable insofar as the associations between populations and their languages are

276 generally quite stable. However, such detailed trees can generally only be constructed

277 for a small number of closely related populations. Super-trees [64], which patch together

278 multiple of these trees into a global phylogeny [65], offer the potential to explore the

279 generality of explanatory hypotheses for cultural diversity, but they may incorporate very

280 different histories in different parts of the tree. These differences could reflect the distinct

281 data sources from each branch of the tree, or genuine lineage-specific evolutionary 
282 patterns, to which we turn below. It is also important to consider whether the trees chosen

283 to represent population histories reflect a time scale appropriate to the social learning and

284 transmission processes that generated the data being evaluated by the comparative 285 method.

287 (ii) Are there lineage-specific or adaptive effects where trait changes differ in different 288 parts of the sample?

289 Traits may show different evolutionary patterning in different language families and/or in 290 different parts of the world. Take, for example, post-marital residence (whether a new 291 married couple live with his, her or neither set of parents). Given the range of ecological, 292 technological, and institutional factors that have been shown to influence this cultural 293 pattern, it is hardly surprising that there are contradictory findings between different 294 studies conducted on different samples [as discussed in 50,66]. These can usefully be 295 thought of as lineage-specific effects with respect to the patterning of evolutionary 296 transitions [49]. As long as the sample sizes in each part of the sample are sufficient for 297 inference, this diversity should be seen as a strength of phylogenetically-based global 298 comparisons, providing material with which to sharpen our understanding of the precise 299 conditions under which particular evolutionary transitions occur. In short while there may 300 be a justification for aiming to test classical hypotheses at increasingly global scales [e.g., 30160 ] failure to find generalized support may actually be highly informative. In similar vein 302 we might expect to find different evolutionary dynamics in agricultural versus foraging 303 societies, rendering problematic inferences made from current samples that are typically 
304 dominated by agriculturalists, who (as in our opening metaphor) offer only a limited 305 palette.

307 In cases where we know or suspect the specific factors that influence cultural patterns 308 differently across the tree, we can be more explicit about evolutionary processes. For 309 example, Ross et al. [67] first built an explicit evolutionary model to assess how the 310 classification of societies as stratified or not might influence the origin and maintenance

311 of the practice of female genital modification. Next, they used an approach based on the

312 Ornstein-Uhlenbeck process [68] which allows for adaptive hypotheses to be evaluated

313 with phylogenetic models that explicitly include adaptive dynamics, both to test for

314 coevolution across the phylogeny and to contrast the strength of selection on a trait with

315 that of drift and other selective processes. In addition to testing explicit co-evolutionary

316 hypotheses, external knowledge about the influence of a factor on the cultural trait can

317 also help to reduce the error in reconstruction of the history of the trait.

319 (iii) Are inferences affected by the resolution and structure of the tree?

320 Phylogenetic relationships among an increasing number of societies are starting to be 321 described, primarily based on language patterns [6], and increasingly incorporated into 322 supertrees [e.g., 69]. Details of the phylogeny are important to understanding how much 323 information about evolutionary processes can be extracted [70]. One issue is uncertainty 324 in the tree: researchers need to consider carefully whether their tree provides a clear 325 branching pattern, as well as robust dates for when the splits occurred. Analyses can now 326 incorporate uncertainty, but often at a reduction in the power to make inferences [71]. In 
327 addition, the shape and the size of the phylogeny influence the inferences that can be

328 made. Small sets of societies, which branch off very early, can become very influential

329 for the reconstruction of internal nodes, the same way that outgroups are used to

330 reconstruct the state of a common ancestor of a clade [72], if data are available for only

331 few societies in the main clade. More generally, balanced trees, with regular branching

332 patterns across the tree, appear more robust to phylogenetic inferences of cultural traits,

333 especially in the presence of horizontal transmission, than unbalanced trees [73]. In

334 contrast, phylogenies with early bursts of speciation, with long branches leading to the

335 societies, contain less information to extract the correct patterns of changes in cultural 336 traits [70].

338 (d) Is this the right sample from which to make inferences?

339 (i) Is the sample large enough?

340 Finding societies, or ethnolinguistic units, with appropriate measures for a cultural

341 evolutionary analysis can be challenging. The vast majority of research to date is based

342 on language families with well-resolved trees, although the number of societies available

343 with appropriate data is often quite small. So, for example, in a recent study of post-marital

344 residence Indo-European provided 27 societies, Uto-Aztecan 26 societies, Pama-

345 Nyungan 66 societies, Bantu 69 societies, and Austronesian 135 societies [49]. In a

346 different kind of study investigating the association between parasite stress and political

347 traits like authoritarianism and democracy data were available only from 52 nation states

348 [30]. The issue is not just the number of societies with data (tips), but the total number of

349 societies in a clade, and the patterning of variation among them: if variant $A$ is present in 
350 all societies in one clade and trait B present in all societies of the other clade, most likely

351 only one change occurred, making it impossible to associate any factor with that change

352 statistically. Indeed, Moravec et al [49] had to drop the entire Indo-European family from

353 their study because there were no matrilocal populations. The obverse (too many

354 changes) is also a problem: if each society differs from its neighbour, it is impossible to

355 reconstruct changes in the past - in our metaphor, the picture of the past is murky brown.

356 In summary, available sample sizes may not allow for reliable inferences.

358 (ii) What about missing data and extinction?

359 Researchers might have to drop cases from their phylogeny, or sample societies 360 strategically, on the basis of trait data availability. Missing data are an issue for at least

361 two reasons. First, if information is available for many societies in one clade but only a

362 few societies in other clades, trees will be highly unbalanced (see Section 2ciii). Second,

363 data (or branches) may be missing not at random with respect to the trait of interest. With

364 respect to the first issue, in Hrnčiř et al's study [38] the sample for studying the association

365 between dwelling size and post-marital residence was heavily biased to New World sites,

366 on account of the scant attention payed to material traits (such as house size) by

367 European as opposed to American ethnographers. While the resulting sample may not

368 be biased with respect to the hypothesis (i.e., data are missing at random) the effect of

369 such uneven and sparse sampling might obscure any phylogenetic signal in the variables

370 and influence inferences about internal nodes. With regard to the second problem, where

371 there is an absence of records (e.g., among foragers in highly productive habitats, see 
372 below), inferences must be drawn with great caution because, following our metaphor,

373 we simply do not have the full palette of colours.

374

375 Extinctions, including unknown ones, are a linked concern insofar as simulation studies

376 show that high extinction rates bring considerable error to estimates of root states [45],

377 as well as influence Type I and II errors [31-33]. Furthermore, extinctions are unlikely to

378 be random. The reason a society is extinct (i.e., does not appear at a tip) may well be

379 associated with its trait values prior to extinction. For example, examining prevalent

380 marriage customs (e.g., are they arranged, are they polygynous) on a tree of

381 contemporary hunter-gatherers [74] may bias ancestral estimates toward the traits of

382 populations that have managed to persist, often in highly marginal areas, into the present

383 era. Suroweic et al [50] reconstructed, both in their global super-tree and in the Bantu

384 language family, the ancestral state for lineality to be non-matrilineal and the ancestral

385 state for post-marital residence to be non-matrilocal [in the case of Bantu contrary to

386 inferences from historical and linguistic data, 75] Could this be because matrilineal (and

387 matrilocal) societies are less likely to have survived shifts to intensive agriculture and 388 pastoralism, which typically favour male-centred kinship institutions?

\section{Assessing potential challenges through simulation}

391 (a) Simulations to reveal both general and specific limitations

392 Various studies have used simulations of the likely processes that generate and modify

393 traits to assess the challenges discussed above. These typically focus on the general

394 feasibility of cultural macroevolution studies [e.g., 31, 45] or the limitation of particular 
395 approaches to recover the history of traits [e.g., 70, 76, 77]. Here, following previous

396 suggestions [e.g., 16, 34], we show that simulations can be usefully employed to assess

397 the extent to which these challenges might influence a particular study.

399 Our simulations investigate the potential impact that the different challenges might have 400 on phylogenetic reconstructions of trait evolution. We focus on three types of inferences 401 that are common in studies of the phylogenetic reconstruction of trait evolution (Figure 1): 402 are some changes between the states of the trait favoured (inference i)?; do 403 socioecological conditions influence which changes among the states of the trait occur 404 (inference ii)?; are there differences between lineages in which changes among the states 405 of the trait occur (inference iii)? In trying to address these inferences, there is a risk of 406 concluding that the answer to the inference is yes even though in truth there was no effect 407 (false positive Type I error) and of concluding that the answer to the inference is no even 408 though there was an effect (false negative Type II error). Our simulations estimate the 409 expected chance of obtaining false positive or false negative conclusions given a 410 particular phylogeny and dataset. In addition, following from the discussion above, we 411 examine how these error rates are influenced by properties of the sample (sample size, 412 missing data), properties of the tree (tree shape), and properties of the traits (rate of 413 change, number of variants, horizontal transmission) (Figure 1). By simulating likely 414 histories of the trait of interest across the given phylogeny, the power to discern alternative 415 histories can be assessed. 
417 We demonstrate this approach using two language phylogenies, one for societies in in 418 the Western North American Indian (WNAI) dataset [78] (Figure S1) and one for societies

419 included in a recent phylogenetic study of the Pama-Nyungan language family [79] 420 (Figure S2). For the WNAI, we constructed a phylogenetic tree for the 172 societies based 421 on a hierarchical language classification [80, modified by 81$]$. For analyses that require 422 bifurcating trees, we resolved polytomies in the phylogenies randomly, but did not 423 represent this uncertainty by repeating the simulations across multiple resolutions 424 because our aim here is to illustrate example cases. For the 306 Pama-Nyungan 425 societies, we used the phylogeny provided in the study. The results we present are 426 dataset-specific, so inferences cannot be directly transferred to other studies. However, 427 several of the findings are consistent across the two phylogenies, and we emphasize we 428 selected language trees that largely reflect the sample size, tree shape and trait 429 distributions that cultural evolutionists typically encounter. We provide all input data, code 430 and output (https://github.com/dieterlukas/CulturalMacroevolution Simulation). The code 431 can be adapted for use on any phylogeny prior to analysis to assess the specific power 432 of a given dataset to test phylogenetic hypotheses. 
434 We focus on three inferences about the evolution of cultural traits, and the errors that can occur when trying to address 435 them.

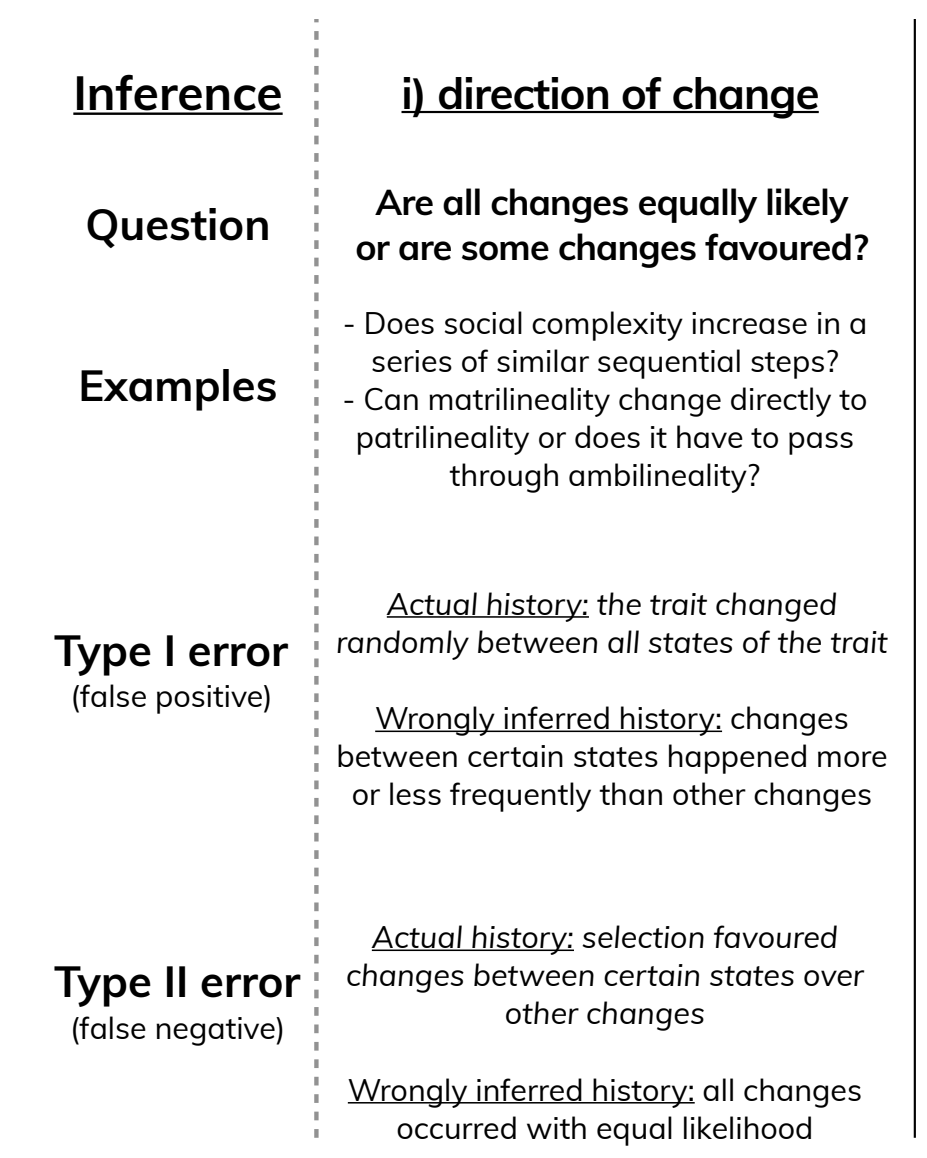

\section{ii) socioecological influences}

Do socioecological conditions influence which changes occur?

- Does the presence of social stratification influence whether female genital modification practices occur? - Does subsistence mode influence sex biases in residence patterns?

Actual history: changes in the trait did not depend on the ecological conditions

Wrongly inferred history: ecological conditions influenced changes between certain states to occur more frequently

Actual history: Changes to certain states were more common in some ecological conditions than others

Wrongly inferred history: changes did not depend on ecological conditions

\section{iii) lineage differences}

Are there differences between lineages in which changes occur?

- Does the pathway from patrilineality to matrilineality differ in different lineages?

- Are changes in grammatical features universal or language-dependent?

Actual history: changes in the trait did not differ between different parts of the phylogenetic tree

Wrongly inferred history: changes between certain states happened more or less frequently than other changes

Actual history: Changes to certain states are more common in some parts of the tree

Wrongly inferred history: changes occurred equally in all parts of the tree 


\section{7 (b) Methods for our simulations of cultural macroevolution}

438 (i) Simulating discrete and continuous traits

439 Across the two phylogenies, we simulated the evolution of discrete (having either two or

440 four different states) and continuous traits using functions of the package 'phytools' in R

441 [82]. Simulations start at the root (with the first discrete variant or the value zero for

442 continuous traits), and estimate transitions along all branches. Transitions between

443 different states (discrete traits) or rates of change (continuous traits) occurred according

444 to four different scenarios. In the first scenario (to obtain the rate of Type I error rates of

445 wrongly inferring directional changes, of wrongly inferring socioecological influences on

446 transitions, and of wrongly inferring lineage differences), all transitions and changes were

447 the same and occurred randomly and with one consistent probability, reflecting a null

448 model drift scenario, where all changes are equally likely across the whole tree. In the

449 second scenario (to obtain the Type II error rate of wrongly missing directional change),

450 there are two rates of transitions and changes. For discrete traits, one rate reflects higher

451 transitions towards one of the variants from the other one or three variants whereas the

452 other rate reflects all other transitions. For continuous traits, increases are more likely

453 than decreases. In the third scenario (to obtain the Type II error rate of wrongly missing

454 socioecological differences), transition rates differ between societies associated with

455 different socioecologies, such that one variant (discrete traits) or positive values

456 (continuous traits) are favoured in branches within lineages associated with different

457 socioecologies. To represent different socioecological conditions as potential correlates

458 of the changes in the simulated cultural trait, we classified the WNAI societies as either

459 living in forest habitats or not, and the Pama-Nyungan societies as hunter-gatherers or 
460 food-producers. In the fourth scenario, transition rates differ between lineages, with 461 changes towards one of the variants (discrete traits) or increases towards positive values

462 (continuous traits) more common along the branches of the largest clade compared to

463 the rest of the tree (to obtain the Type II error rate of wrongly missing lineage differences).

465 For all scenarios, we extract the simulated states of the trait across the tips of the phylogeny. The reported error rates reflect the number of independent simulations in

467 which the analysis supported the wrong inference. The error rates for the baseline (Figure

$4682 \mathrm{~A})$ are based on 110 simulations each, reflecting the different settings and ten

469 independent repetitions of each setting. The error rates for simulations assessing the

470 influence of properties of the sample, of the tree, and of the trait are based on the

471 respective subsets of simulations, and we report how these deviations from our chosen

472 baseline change the proportion of wrong inferences (indicated by + and - designations in

473 Figure 2B-D and Supplementary Figures S3-S5).

474

\section{Figure 2: Sources and rates of errors across the simulations of trait evolution for} the WNAI and the Pama-Nyungan societies

The illustrations show examples of potential sources of errors in phylogenetic reconstructions of trait evolution. They depict the evolutionary history of a trait with two states (red and black, representing either a discrete trait or negative and positive values of a continuous trait), the resulting values at the tip, and most likely reconstruction. The values underneath show the error rates in baseline simulations (A), and how much these change (increase + or decrease -) depending on an example property of the sample (missing data, B), an example property of the trait (random horizontal transmission, C), and an example property of the tree (a late-burst phylogeny, D). The reported error rates display the percentage of independent repetitions in which a phylogenetic reconstruction of trait evolution either wrongly supported an evolutionary model in which rates of change differed across the tree even though all changes were simulated to occur randomly (Type I error, false positive); or wrongly did not support an evolutionary model in which rates of change differed even though this was how the change was simulated (Type II error, false negative). 


\section{A) Baseline simulation}

tree is undated for WNAl, dated for Pama-Nyungan

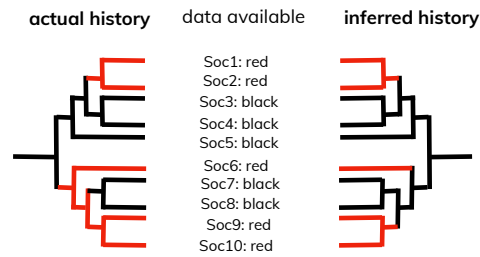

With a standard approach, inferences are probabilistic, and might occasionally lead to errors deeper in the tree

error rates discrete trait

\begin{tabular}{|c|c|c|c|c|c|}
\hline \multirow{2}{*}{\multicolumn{2}{|c|}{$\begin{array}{l}\text { direction of change } \\
\text { Type l: false positive }\end{array}$}} & \multicolumn{2}{|c|}{ socioecological influences } & \multicolumn{2}{|c|}{ lineage differences } \\
\hline & & \multicolumn{2}{|c|}{ Type I: false positive } & \multicolumn{2}{|c|}{ Type I: false positive } \\
\hline $\begin{array}{l}\text { WNAI } \\
12 \%\end{array}$ & $\begin{array}{l}\text { PamaNy } \\
15 \%\end{array}$ & $\begin{array}{l}\text { WNAI } \\
40 \%\end{array}$ & $\begin{array}{l}\text { PamaNy } \\
29 \%\end{array}$ & $\begin{array}{l}\text { WNAI } \\
38 \%\end{array}$ & $\begin{array}{l}\text { PamaNy } \\
26 \%\end{array}$ \\
\hline \multicolumn{2}{|c|}{ Type II: false negative } & \multicolumn{2}{|c|}{ Type II: false negative } & \multicolumn{2}{|c|}{ Type II: false negative } \\
\hline $\begin{array}{l}\text { WNAI } \\
52 \%\end{array}$ & $\begin{array}{l}\text { PamaNy } \\
36 \%\end{array}$ & $\begin{array}{l}\text { WNAI } \\
65 \%\end{array}$ & $\begin{array}{l}\text { PamaNy } \\
46 \%\end{array}$ & $\begin{array}{l}\text { WNAI } \\
54 \%\end{array}$ & $\begin{array}{l}\text { PamaNy } \\
60 \%\end{array}$ \\
\hline \multicolumn{6}{|c|}{ error rates continuous trait } \\
\hline \multirow{2}{*}{\multicolumn{2}{|c|}{$\begin{array}{l}\text { direction of change } \\
\text { Type I: false positive }\end{array}$}} & \multicolumn{2}{|c|}{ socioecological influences } & \multicolumn{2}{|c|}{ lineage differences } \\
\hline & & \multicolumn{2}{|c|}{ Type I: false positive } & \multicolumn{2}{|c|}{ Type l: false positive } \\
\hline $\begin{array}{l}\text { WNAI } \\
13 \%\end{array}$ & $\begin{array}{l}\text { PamaNy } \\
0 \%\end{array}$ & $\begin{array}{l}\text { WNAl } \\
15 \%\end{array}$ & $\begin{array}{l}\text { PamaNy } \\
51 \%\end{array}$ & $\begin{array}{l}\text { WNAI } \\
6 \%\end{array}$ & $\begin{array}{l}\text { PamaNy } \\
48 \%\end{array}$ \\
\hline \multicolumn{2}{|c|}{ Type II: false negative } & \multicolumn{2}{|c|}{ Type II: false negative } & \multicolumn{2}{|c|}{ Type Il: false negative } \\
\hline $\begin{array}{l}\text { WNAI } \\
42 \%\end{array}$ & $\begin{array}{l}\text { PamaNy } \\
51 \%\end{array}$ & $\begin{array}{l}\text { WNAI } \\
1 \%\end{array}$ & $\begin{array}{l}\text { PamaNy } \\
2 \%\end{array}$ & $\begin{array}{l}\text { WNAI } \\
0 \%\end{array}$ & $\begin{array}{l}\text { PamaNy } \\
0 \%\end{array}$ \\
\hline
\end{tabular}

\section{C) Example of property of the trait} random horizontal transmission actual history data available inferred history

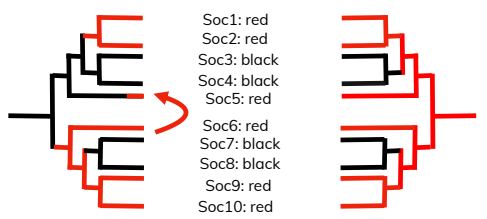

A high rate of independent horizontal transmission will introduce outliers into clades: for rare binary traits this can lead to spurious associations while for continuous traits it will obscure patterns

\section{error rates discrete trait}

\begin{tabular}{|c|c|c|c|c|c|}
\hline \multirow{2}{*}{\multicolumn{2}{|c|}{$\begin{array}{l}\text { direction of change } \\
\text { Type I: false positive }\end{array}$}} & \multirow{2}{*}{\multicolumn{2}{|c|}{ socioecological influences }} & \multicolumn{2}{|c|}{ lineage differences } \\
\hline & & & Type l: false positive & \multicolumn{2}{|c|}{ Type I: false positive } \\
\hline $\begin{array}{l}\text { WNAI } \\
+39 \%\end{array}$ & $\begin{array}{c}\text { PamaNy } \\
+31 \%\end{array}$ & $\begin{array}{l}\text { WNAI } \\
+1 \%\end{array}$ & $\begin{array}{l}\text { PamaNy } \\
+18 \%\end{array}$ & $\begin{array}{l}\text { WNAI } \\
+1 \%\end{array}$ & $\begin{array}{l}\text { PamaNy } \\
+15 \%\end{array}$ \\
\hline \multicolumn{2}{|c|}{ Type II: false negative } & \multicolumn{2}{|c|}{ Type II: false negative } & \multicolumn{2}{|c|}{ Type II: false negative } \\
\hline $\begin{array}{l}\text { WNAl } \\
-21 \%\end{array}$ & $\begin{array}{c}\text { PamaNy } \\
-13 \%\end{array}$ & $\begin{array}{l}\text { WNA } \\
-3 \%\end{array}$ & $\begin{array}{c}\text { PamaNy } \\
-1 \%\end{array}$ & $\begin{array}{l}\text { WNAI } \\
-20 \%\end{array}$ & $\begin{array}{c}\text { PamaNy } \\
-18 \%\end{array}$ \\
\hline \multicolumn{6}{|c|}{ error rates continuous trait } \\
\hline \multicolumn{2}{|c|}{ direction of change } & \multicolumn{2}{|c|}{ socioecological influences } & \multicolumn{2}{|c|}{ lineage differences } \\
\hline \multicolumn{2}{|c|}{ Type I: false positive } & \multicolumn{2}{|c|}{ Type I: false positive } & \multicolumn{2}{|c|}{ Type l: false positive } \\
\hline $\begin{array}{l}\text { WNAI } \\
+77 \%\end{array}$ & $\begin{array}{c}\text { PamaNy } \\
+78 \%\end{array}$ & $\begin{array}{l}\text { WNA } \\
-8 \%\end{array}$ & $\begin{array}{c}\text { PamaNy } \\
-48 \%\end{array}$ & $\begin{array}{l}\text { WNAI } \\
+28 \%\end{array}$ & $\begin{array}{l}\text { PamaNy } \\
+21 \%\end{array}$ \\
\hline \multicolumn{2}{|c|}{ Type II: false negative } & \multicolumn{2}{|c|}{ Type Il: false negative } & \multicolumn{2}{|c|}{ Type II: false negative } \\
\hline $\begin{array}{l}\text { WNAI } \\
-30 \%\end{array}$ & $\begin{array}{l}\text { PamaNy } \\
-26 \%\end{array}$ & $\begin{array}{l}\text { WNA } \\
+94 \%\end{array}$ & $\begin{array}{l}\text { PamaNy } \\
+95 \%\end{array}$ & $\begin{array}{l}\text { WNAI } \\
+1-0 \%\end{array}$ & $\begin{array}{l}\text { PamaNy } \\
+/-0 \%\end{array}$ \\
\hline
\end{tabular}

B) Example of property of the sample data is missing for one variant

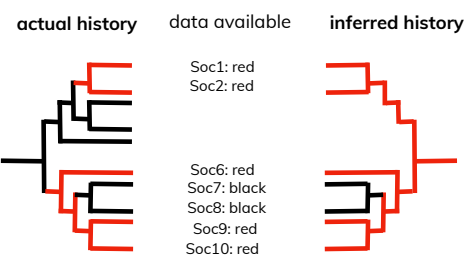

If there are biases in which data are available, it might lead to wrong inferences of internal nodes and reduces power to detect associations

$$
\begin{aligned}
& \text { error rates discrete trait } \\
& \begin{array}{ccc}
\text { direction of change } & \text { socioecological influences } & \text { lineage differences } \\
\text { Type l: false positive } & \text { Type l: false positive } & \text { Type I: false positive }
\end{array} \\
& \text { WNAl Pamany WNAl Pamany WNAl PamaNy }
\end{aligned}
$$

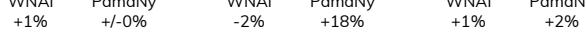

$$
\begin{aligned}
& \text { Type II: false negative Type II: false negative Type Il: false negative } \\
& \text { WNAl Pamany WNAl Pamany WNAl Pamany } \\
& \text { error rates continuous trait }
\end{aligned}
$$

\begin{tabular}{|c|c|c|c|c|c|}
\hline \multicolumn{6}{|c|}{ or ra } \\
\hline \multicolumn{2}{|c|}{ direction of change } & \multicolumn{2}{|c|}{$\underline{\text { socioecological influences }}$} & \multicolumn{2}{|c|}{ lineage differences } \\
\hline \multicolumn{2}{|c|}{ Type l: false positive } & \multicolumn{2}{|c|}{ Type I: false positive } & \multicolumn{2}{|c|}{ Type l: false positive } \\
\hline $\begin{array}{l}\text { WNAI } \\
-4 \%\end{array}$ & $\begin{array}{c}\text { PamaNy } \\
-3 \%\end{array}$ & $-21 \%$ & +4 & $\begin{array}{l}\text { WNAI } \\
+18 \%\end{array}$ & $\begin{array}{l}\text { PamaNy } \\
+7 \%\end{array}$ \\
\hline \multicolumn{2}{|c|}{ Type II: false negative } & \multicolumn{2}{|c|}{ Type II: false negative } & \multicolumn{2}{|c|}{ Type II: false negative } \\
\hline $\begin{array}{l}\text { WNA } \\
+13 \%\end{array}$ & $\begin{array}{c}\text { PamaNy } \\
+15 \%\end{array}$ & $\begin{array}{l}\text { WNAI } \\
+28 \%\end{array}$ & & $\begin{array}{c}\text { WNAI } \\
-8 \%\end{array}$ & \\
\hline \multicolumn{6}{|c|}{ error rates continuo } \\
\hline \multicolumn{2}{|c|}{ direction of change } & \multicolumn{2}{|c|}{$\underline{\text { socioecological influences }}$} & \multicolumn{2}{|c|}{ lineage differences } \\
\hline \multicolumn{2}{|c|}{ Type l: false positive } & \multicolumn{2}{|c|}{ Type I: false positive } & \multicolumn{2}{|c|}{ Type l: false positive } \\
\hline $\begin{array}{l}\text { WNAI } \\
+46 \%\end{array}$ & $\begin{array}{c}\text { PamaNy } \\
+62 \%\end{array}$ & $\begin{array}{l}\text { WNAI } \\
+1-0 \%\end{array}$ & $\begin{array}{c}\text { PamaNy } \\
-2 \%\end{array}$ & $\begin{array}{l}\text { WNAI } \\
+34 \%\end{array}$ & $\begin{array}{c}\text { PamaNy } \\
+13 \%\end{array}$ \\
\hline \multicolumn{2}{|c|}{ Type II: false negative } & \multicolumn{2}{|c|}{ Type II: false negative } & \multicolumn{2}{|c|}{ Type Il: false negative } \\
\hline $\begin{array}{l}\text { WNAI } \\
-6 \%\end{array}$ & $\begin{array}{l}\text { PamaNy } \\
-23 \%\end{array}$ & $\begin{array}{l}\text { WNAI } \\
+90 \%\end{array}$ & $\begin{array}{c}\text { PamaNy } \\
+60 \%\end{array}$ & $\begin{array}{l}\text { WNA } \\
+1-0 \%\end{array}$ & $\begin{array}{c}\text { PamaNy } \\
+/-0 \%\end{array}$ \\
\hline
\end{tabular}

\section{D) Example of property of the tree}

late-burst phylogeny with divergent lineages

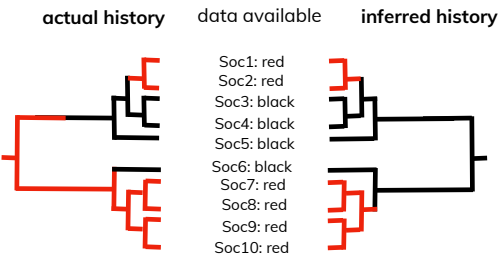

Lineages will show differences by chance if they are separated by long branches, and if variation in ecology arose in all lineages short branches do not provide sufficient time for selection to lead to a response 
493 To determine whether some changes in the simulated discrete traits occurred more

494 frequently than other changes (inference i), we compare the likelihood of a reconstruction

495 in which all transitions are assumed to occur at the same rate to that of a reconstruction

496 in which some transitions occur more frequently than others. We estimate the likelihoods

497 of the equal and the biased models using the function 'ace' of the package 'ape' [83] in

$498 \mathrm{R}$, assessing significance with a likelihood ratio test adjusting the degrees of freedom to

499 reflect the different number of parameters in the two models. To determine whether some

500 changes occurred more in specific socioecological conditions (inference ii), we compare

501 the likelihood of a reconstruction where transitions in the simulated trait are independent

502 from changes in the socioecological condition to a reconstruction where changes in the 503 simulated trait depend on the socioecological condition. We used the function 'discrete'

504 in the software Bayestraits [84] to estimate the likelihood of a dependent model and the 505 likelihood of an independent model, comparing them using a likelihood ratio test. To 506 determine whether changes were more likely to have occurred in the largest clade 507 compared to the rest of the tree (inference iii), we estimate the likelihood of a 508 reconstruction where transitions in the simulated trait are independent of where they 509 occur in the tree and the likelihood of a reconstruction where transitions in the simulated 510 trait are dependent on whether they occur on a branch in the largest clade or on a branch

511 in a different part of the tree. For this we also used the function 'discrete' in the software 512 Bayestraits and conducted a likelihood ratio test. 
514 To determine whether changes in the simulated continuous traits occurred predominantly

515 in one direction rather than randomly (inference i), we compared the likelihood of a

516 reconstruction in which changes occurred as under a null Brownian drift model to a

517 reconstruction in which changes occurred with a trend in one direction using functions of

518 the package 'OUwie' [85] in $\mathrm{R}$, assessing significance with a likelihood ratio test. To

519 determine whether values differed between societies in different socioecological

520 conditions (inference ii), we performed phylogenetic regressions to assess whether

521 socioecological conditions are associated with differences in the simulated values

522 observed at the tips [using functions of the package 'MCMCglmm' in R, 86], assessing

523 significance using the p-value). To determine whether values in the largest clade differed

524 from the values in the remainder of the tree, we performed phylogenetic regressions to

525 compare all values in the largest clade to the remaining values (using functions of the

526 package 'MCMCglmm' in R, and assessing significance using the $p$-value).

528 (iii) Identifying the effect of differences in the properties of the trait, of the sample, and of 529 the tree

530 In addition to a baseline scenario, we varied the following parameters during the 531 simulations: properties of the trait, of the sample, and of the tree. To change properties

532 of the trait we: a) set the number of possible variants of the trait to be either two or four

533 (discrete traits), b) changed the rate at which transitions could occur from low to high (for

534 a slow rate, this equals to an average of 10 changes across the 610 branches in the

535 Pama-Nyungan phylogeny and about half as many changes across the 342 branches of 536 the WNAI phylogeny; for a fast rate, this equals to an average of 75 changes across the 
537 Pama-Nyungan phylogeny and about 40 changes across the WNAI phylogeny), and c)

538 introduced horizontal transmission by having $10 \%$ societies at the tips copy a variant of

539 another random society, another society in the same ecology, or their closest geographic

540 neighbour. To change properties of the sample, we investigated samples with: a) the full

541 set of societies, b) only societies in the largest clade, and c) $25 \%$ or $50 \%$ of data missing

542 from a random set of societies, from societies in the dominant ecology, or from societies

543 with one of the variants (discrete traits) or with positive trait values (continuous traits). To

544 change properties of the tree, we manipulated branch lengths: a) to reflect trait change at

545 the time of split, where branches lengths are all the same, b) to reflect an early burst into

546 the existing societies, where branches between clades are short and branches to the tips

547 are long, and c) to reflect a late radiation where branches separating clades are long and

548 all diversification within the clades happened very recently. We display examples of

549 manipulations of properties of the trait, of the sample, and of the tree in Figure 2 and 550 below; for additional results see Supplementary Materials.

552 (c) Findings from our simulations of cultural macroevolution

554 Our simulations show that for these particular datasets and phylogenies, properties of the 555 trait have the biggest influence on the frequency of erroneous inferences, properties of 556 the sample have a relatively minor influence, while the influence of properties of the tree 557 are context-specific (Figure 2). 
559 We set up the baseline simulations to generate data similar to those commonly available

560 in cultural phylogenetics, and we analyzed the simulated data with the standard settings

561 of the respective methods. In these baselines, we obtained substantial false positive

562 (Type I) and false negative (Type II) errors that range between $12 \%-40 \%$ and $36 \%-65 \%$

563 respectively for discrete traits, and $0 \%-51 \%$ and $0 \%-51 \%$ for continuous traits (Figure

$5642 \mathrm{2}$ ). Continuous traits generally show lower false negative errors than discrete traits: the

565 amount of variation in discrete traits is restricted, reducing power to detect weak effects,

566 in particular when relying on the standard settings of the analyses which attempt to

567 estimate a large number of parameters without any prior information. False positive rates

568 are largely comparable for continuous and discrete traits: the simulated traits changed

569 relatively rapidly, such that by chance lineages and ecologies will differ. There is no

570 consistent difference in performance between the two language trees, despite their

571 difference in shape and tree structure balance. In the following, we present changes in

572 the errors contingent on various properties of traits, trees and sample, but we should

573 emphasize that throughout errors are high. While undoubtedly some of these problems

574 might be specific to the situations we chose to simulate, and the precise magnitude of the

575 errors might be reduced by adapting the settings of the analyses to independently

576 available information (such as likely types of transmission), the size of these errors should

577 not be overlooked.

579 Regarding directional change, the false positive error of wrong inference was was

580 increased most markedly from the baseline rate when variants of the trait were 581 transmitted horizontally among societies (Figure 2C, Figure S3). Such horizontal 
582 transmission might reflect actual directional changes, but we found relatively high error 583 rates even when horizontal transmission occurred randomly: relatively high rates of 584 random horizontal transmission (in our simulations $10 \%$ of extant societies copy a variant) 585 will introduce highly divergent variants, leading to Type I errors of wrongly supporting a 586 positive signal as these divergent values would require faster changes than assumed 587 under a null model of random drift. The Type II error of wrongly missing directional change was generally high both for discrete and continuous traits: it was highest when power was

589 reduced because there are very few changes in total across the phylogeny, that is with 590 fewer variants of the trait (Figure S5) and lower when horizontal transmission increased 591 the frequency of certain states throughout the tree (Figure 2C).

593 Regarding the detection of socioecological influences on change, although the false 594 positive error rate was already high in the baseline for both discrete and continuous traits, 595 it was larger when there was horizontal transmission that could increase the occurrence 596 of a rare variant within one ecology (Figure S4) and when the societies in different 597 ecologies are separated by long branches such that changes might occur by chance (e.g. 598 late burst phylogeny for the Pama-Nyungan, Figure 2D). The false negative error rate of 599 missing socioecological influences on changes was highest when data was missing non600 randomly (Figure 2B), in particular if certain variants are rare to begin with (less than $10 \%$ 601 of Pama-Nyungan societies are food-producers, Figure S2). False negative error rates 602 for detecting a socioecological influence also increased under random horizontal 603 transmission (Figure 2C) and late-burst phylogenies (Figure 2D) in both language 604 families, although only for continuous traits. 
606 Regarding lineage-specific patterns, the false positive error rate of wrongly inferring

607 lineage differences on changes was already high in the baseline simulation for both

608 discrete and continuous traits. It was considerably increased when there was structured

609 horizontal transmission within lineages or ecologies (Figure S3), random horizontal

610 transmission (Figure 2C), and as a result of tree structure, specifically in late-burst

611 phylogenies where long branches separate lineages such that differences might occur by

612 chance (Figure 2D). The false negative error rate of wrongly missing lineages differences

613 was already high in the baseline (for discrete traits) and appears to decline when

614 horizontal transmission occurs (Figure 2C, Figure S3).

\section{4. Discussion and Outlook}

617 The most striking aspect of these simulations is the very high rate of false positive Type I

618 and false negative Type II errors contingent on properties of the traits, of the tree shape,

619 and less so of the sample. While this result may be specific to the WNAI dataset (with its

620 poorly resolved language phylogeny, its categorical codings, and its unbalanced clade

621 structures), largely similar false positive and false negative errors in the Pama-Nyungan

622 analyses suggest they might be quite general.

623

624 The strong effects of horizontal transmission on false inferences about selection may 625 reflect the fact that in the WNAI linguistic and spatial signals are relatively uncorrelated 626 [26, see also 39]. This is not always the case; phylogenies vary in the extent to which they 627 can reliably capture the spread of cultural traits, which will depend on whether or not there 
628 has been a well-documented linguistic and cultural expansion as in the Austronesian and

629 Bantu language families. While we might all hope that the WNAI-specific findings are not

630 generalizable, the analysis of Pama-Nyungan (with its better-balanced clade structure) is

631 concerning in that it shows very similar false inferences resulting from horizontal

632 transmission.

633

634 Despite the fact that cultural phylogeneticists differentiate, quite rightly, the distinct 635 objectives of different cultural phylogenetic studies, our simulation results reveal problems 636 that are not particular to any one objective. More specifically, we show that properties of 637 the traits, tree and (less so) sample generate inferential problems that result from 638 inaccurate reconstructions of internal nodes. These inaccuracies impact not just studies 639 that seek determination of ancestral states, but also those concerned with the 640 identification of independent adaptations, the understanding of potential sequences of 641 cultural transitions, and the determination of whether transitions are correlated with 642 changes in other traits, insofar as each of these objectives requires estimation of internal 643 nodes.

645 Given the potential generality of these effects of properties of traits (and to a lesser extent 646 of the tree and the sample) on the validity of inferences, and the relevance of these 647 findings for the multiple objectives within cultural phylogenetic research, we suggest that 648 the concerns we review in Section 2 continue to be legitimate. Accordingly, we offer a 649 simulation that identifies the expected error rates associated with a large set of trait, tree 650 and sample properties (see code on GitHub) which we encourage investigators to use to 
651 determine the error rates they might expect in their particular analysis. We view our

652 contribution here as complementary to that of staunch supporters of phylogenetic

653 analyses who have themselves acknowledged that, where cultural reticulation has been

654 rampant, projecting traits onto trees can only produce "meaningless results" [34, page

$655366]$, adding that phylogenetic analysis may not be suitable for all temporal and spatial

656 time scales [3].

657

658 We also propose the following items as a starting point for a checklist when planning a

659 cultural macroevolution study. (1) Research questions should be informed by solid

660 theoretical models [e.g., 67, 87]. This will help address many of the dilemmas and

661 tradeoffs outlined here - specifically with respect to how to identify which sample to use,

662 and how to measure the trait of interest. (2) Investigators should consider carefully

663 whether the tree chosen to represent population histories reflects a time scale appropriate

664 to the social learning and transmission processes that generated the data being evaluated

665 by the comparative method. Ideally, the tree will reflect diverse sources of data across

666 history, linguistics, genetics and archaeology [see also 41]. (3) Investigators should

667 consider carefully how the trait of interest is transmitted, using ethnographic or historical

668 materials where available, taking care to differentiate mechanisms of transmission for

669 between and within populations; this will inform about the potential rate of change, and

670 whether horizontal transmission is likely to be prevalent [see also 88]. (4) Investigators

671 should explore how missing data, horizontal transmission, and/or differential lineage

672 extinction might influence their inferences for their particular sample (see our code).

673 Investigators may have additional information about the transmission of the cultural trait 
674 they are studying that can inform the analyses and there are multiple ways to model such

675 evolutionary processes. (5) Investigators should acknowledge the centrality of internal

676 node trait estimation to their inferences. For example, researchers often assert that if they

677 are using phylogenies only to control for statistical independence the precise details of

678 internal node reconstructions are not critical. This may be true, but it is incumbent then

679 not to draw inferences of causality from their phylogenetic analyses when, as we have

680 shown, certain features of the tree, the sample or the traits may exacerbate these

681 inaccuracies.

682

683 To return to our metaphor, we urge investigators to consider carefully what the picture 684 they are painting of the past is actually going to show. While it may not offer perfect 685 resolution, nor the full colour spectrum, is there sufficient detail to discern the key features 686 of interest?

688 In sum, we are not arguing against phylogenetic studies of cultural traits. Rather, we hope 689 our overview and our simulation will encourage researchers to consider persistent perils 690 inherent in answering questions about the history of a cultural trait. While we recognize 691 the importance of the cultural evolutionary goal of putting "pre-history back into 692 anthropology" [11, page 406], we suspect more caution is still needed. Evidence for our 693 position lies in the increasingly contradictory findings that are emerging in the literature 694 regarding ancestral states, sequence of changes, and adaptive value. The very fact that 695 the field has grown enough to produce discrepant findings is positive and generates 696 progress. Discrepancies may well reflect lineage, region or scale-specific effects, as well 
697

698

699

700

701

702

703

704

705

706

707

708

709

710

\section{1}

as differences in the definition or classification of traits which, with further research, will only increase our understanding of the drivers of cultural diversity, but we do need to keep in mind that methodological problems might also be entailed.

\section{Acknowledgments}

We thank the members of the Department of Human Behavior, Ecology \& Culture at the MPI EVA for feedback during the planning of this study, and Fiona Jordan, Sam Passmore and Tom Currie for helpful comments on an earlier version of this manuscript. We also thank the organizers of this special issue, particularly Eva Boon, as well as the Lorentz Center at Leiden University for hosting the Foundations of Cultural Evolution workshop.

\section{References}

[1] Pagel, M. \& Mace, R. 2004 The cultural wealth of nations. Nature 428, 275-278.

[2] Borgerhoff Mulder, M., Nunn, C. L. \& Towner, M. C. 2006 Macroevolutionary studies of cultural trait transmission. Evolutionary Anthropology 15, 52-64.

[3] Gray, R. D. \& Watts, J. 2017 Cultural macroevolution matters. Proceedings of the National Academy of Sciences 114, 7846-7852.

[4] Lipo, C. P., O'Brien, M. J., Shennan, S. \& Collard, M. 2006 Mapping Human History: Culture, Language and Biology. (New Jersey, Aldine Transaction.

[5] Mace, R., Holden, C. J. \& Shennan, S. 2005 The Evolution of Cultural Diversity: A phylogenetic Approach. (London, UCL Press.

[6] Kirby, K. R., Gray, R. D., Greenhill, S. J., Jordan, F. M., Gomes-Ng, S., Bibiko, H.-J., Blasi, D. E., Botero, C. A., Bowern, C., Ember, C. R., et al. 2016 D-PLACE: A Global Database of Cultural, Linguistic and Environmental Diversity. PLOS ONE 11, e0158391. (DOI:10.1371/journal.pone.0158391).

[7] Boas, F. 1940 Race, Language and Culture. New York, The MacMillan Company.

[8] Murdock, G. P. 1949 Social structure. New York, Free Press. 
[9] Moylan, J. W., Borgerhoff Mulder, M., Graham, C. M., Nunn, C. L. \& Hakansson, T. 2006 Cultural traits and linguistic trees: Linguistic signal in East Africa. In Mapping Human History: Phylogenetic Approaches in Anthropology and Prehistory (eds. C. P. Lipo, M. J. O'Brien, S. Shennan \& M. Collard), pp. 33-52. New York, Aldine Transaction. [10] Jordan, P. \& Shennan, S. J. 2003 Cultural transmission, language, and basketry traditions amongst the California Indians. Journal of Anthropological Archaeology 22, 43-74. [11] Mace, R. \& Jordan, F. M. 2011 Macro-evolutionary studies of cultural diversity: a review of empirical studies of cultural transmission and cultural adaptation. Philosophical Transactions of the Royal Society B 366, 402-411. [12] Bortolini, E., Pagani, L., Crema, E. R., Sarno, S., Barbieri, C., Boattini, A., Sazzini, M., da Silva, S. G., Martini, G., Metspalu, M., et al. 2017 Inferring patterns of folktale diffusion using genomic data. Proceedings of the National Academy of Sciences 114, 9140-9145. (DOI:10.1073/pnas.1614395114).

[13] Spolaore, E. \& Wacziarg, R. 2009 The diffusion of development. Quarterly Journal of Economics, 469-529.

[14] Kamilar, J. M. \& Cooper, N. 2013 Phylogenetic signal in primate behaviour, ecology and life history. Philosophical Transactions of the Royal Society B: Biological Sciences 368, 20120341. [15] Blomberg, S. P., Garland, T. \& Ives, A. R. 2003 Testing for phylogenetic signal in comparative data: Behavioral traits are more labile. Evolution 57, 717-745.

[16] McElreath, R. 1997 Iterated parsimony: A method for reconstructing cultural histories [M. Sc. thesis], Department of Anthropology, University of California at Los Angeles.

[17] Lycett, S. J. 2015 Differing patterns of material culture intergroup variation on the high plains: quantitative analyses of parfleche characteristics vs. moccasin decoration. American Antiquity, 714-731.

[18] Boyd, R., Borgerhoff Mulder, M., Durham, W. H. \& Richerson, P. J. 1997 Are cultural phylogenies possible? In Human by Nature: Between Biology and the Social Sciences (eds. P. Weingart, S. D. Mitchell, P. J. Richerson \& S. Maasen), pp. 355-386. Mahwah, NJ, Erlbaum.

[19] Matthews, L. J., Tehrani, J. J., Jordan, F. M., Collard, M. \& Nunn, C. L. 2011 Testing for Divergent Transmission Histories among Cultural Characters: A Study Using Bayesian Phylogenetic Methods and Iranian Tribal Textile Data. PLoS ONE. (DOI:doi:10.1371/journal.pone.0014810).

760 Trends in Ecology and Evolution 13, 356-359.

761 [21] Maddison, W. P. \& Knowles, L. L. 2006 Inferring phylogeny despite incomplete lineage 762 sorting. Systematic Biology 51, 21-30. [22] Verkerk, A. 2019 Detecting non-tree-like signal using multiple tree topologies. Journal of Historical Linguistics p, 9-69.

[23] Tehrani, J. J., Collard, M. \& Shennan, S. 2010 The cophylogeny of populations and cultures: reconstructing the evolution of Iranian tribal craft traditions using trees and jungles. Philosophical Transactions of the Royal Society B-Biological Sciences 365, 3865-3874.

[24] Gray, R. D., Bryant, D. \& Greenhill, S. J. 2010 On the shape and fabric of human history. Phil. Trans. R. Soc. B. 365, 3923-3933. (DOI:doi:10.1098/rstb.2010.0162).

[25] Nakamura, M., Wakano, J. Y., Aoki, K. \& Kobayashi, Y. 2020 The popularity spectrum applied to a cross-cultural question. Theoretical population biology 133, 104-116.

[26] Towner, M. C., Grote, M. N., Venti, J. \& Borgerhoff Mulder, M. 2012 Cultural Macroevolution on Neighbor Graphs: Vertical and Horizontal Transmission among Western North 
American Indian Societies. Human Nature 23, 283-305. (DOI:10.1007/s12110-012-9142-z). [27] Gavin, M. C., Kavanagh, P. H., Haynie, H. J., Bowern, C., Ember, C. R., Gray, R. D., Jordan, F. M., Kirby, K. R., Kushnick, G. \& Low, B. S. 2018 The global geography of human subsistence. Royal Society open science 5, 171897. [28] Beheim, B. A. \& Bell, A. V. 2011 Inheritance, ecology and the evolution of the canoes of east Oceania. Proceedings of the Royal Society B: Biological Sciences 278, 3089-3095. [29] von Cramon-Taubadel, N. \& Lycett, S. J. 2018 Assessing the relative impact of historical divergence and inter-group transmission on cultural patterns: a method from evolutionary ecology. Philosophical Transactions of the Royal Society B: Biological Sciences 373, 20170054. politics: why cross-cultural studies must control for relatedness, proximity and covariation. Royal Society open science 5, 181100. [31] Nunn, C. L., Borgerhoff Mulder, M. \& Langley, S. 2006 Comparative methods for studying cultural trait evolution: A simulation study. Cross-Cultural Research 40, 177-209. [32] Currie, T. E., Greenhill, S. J. \& Mace, R. 2010 Is horizontal transmission really a problem for phylogenetic comparative methods? A simulation study using continuous cultural traits. Philosophical Transactions of the Royal Society B-Biological Sciences. 365, 3903-3912. [33] Nunn, C. L., Arnold, C., Matthews, L. J. \& Borgerhoff Mulder, M. 2010 Simulating Trait Evolution for Cross-Cultural Comparison. Philosophical Transactions of the Royal Society B. Biological Sciences. 365, 3807- 3819.

[34] Gray, R. D., Greenhill, S. J. \& Ross, R. M. 2007 The pleasures and perils of Darwinizing culture (with phylogenies). Biological Theory 2, 360-375.

[35] Rogers, A. R. \& Cashdan, E. 1997 The phylogenetic approach to comparing human populations. Evolution and Human Behavior 18, 353-358.

[36] Borgerhoff Mulder, M. 2001 Using phylogenetically based comparative methods in anthropology: More questions than answers. Evolutionary Anthropology 10, 99-111.

[37] Freckleton, R. P. \& Jetz, W. 2009 Space versus phylogeny: disentangling phylogenetic and spatial signals in comparative data. Proceedings of Royal Society (London) B. 276, 21-30.

[38] Hrnčír, V., Duda, P., Šaffa, G., Květina, P. \& Zrzavý, J. 2020 Identifying post-marital residence patterns in prehistory: A phylogenetic comparative analysis of dwelling size. PLOS ONE 15, e0229363. (DOI:10.1371/journal.pone.0229363).

[39] Sellen, D. W. \& Hruschka, D. J. 2004 Extracted-food resource-defense polygyny in native western North American Societies. Current Anthropology 45, 707-715.

[40] Cashdan, E. \& Rogers, A. R. 1997 Book review of "Human nature: A critical reader". Evolution and Human Behavior 18, 279-283.

[41] Teixidor-Toneu, I., Kool, A., Greenhill, S. J., Kjesrud, K., Sandstedt, J. J., Manzanilla, V. \& Jordan, F. M. this issue Historical, archaeological, and linguistic evidence test the phylogenetic inference of Viking-Age plant use. Phil. Trans. R. Soc Lond. B.

[42] Currie, T. E., Meade, A., Guillon, M. \& Mace, R. 2013 Cultural phylogeography of the Bantu Languages of sub-Saharan Africa. Proceedings of the Royal Society B: Biological Sciences 280, 20130695.

[43] Tëmkin, I. \& Eldredge, N. 2007 Phylogenetics and material cultural evolution. Current Anthropology 48, 146-153.

[44] Burton, M. L., Moore, C. C., Whiting, J. W. M. \& Romney, A. K. 1996 Regions based on social structure. Current Anthropology 37, 87-123. 
[45] Currie, T. E. \& Mace, R. 2014 Evolution of cultural traits occurs at similar relative rates in different world regions. Proceedings of the Royal Society B 281(1795), 20141622. [46] Rogers, D. S. \& Ehrlich, P. R. 2008 Natural selection and cultural rates of change. Proceedings of the National Academy of Sciences 105, 3416-3420. (DOI:doi/10.1073/pnas.0711802105).

824 [47] Jordan, P. 2014 Technology as Human Social Tradition: Cultural Transmission among Hunter Gatherers. Berkeley, University of California Press.

[48] Royston, P., Altman, D. G. \& Sauerbrei, W. 2006 Dichotomizing continuous predictors in multiple regression: a bad idea. Statistics in medicine 25, 127-141.

[49] Moravec, J. C., Atkinson, Q., Bowern, C., Greenhill, S. J., Jordan, F. M., Ross, R. M., Gray, R., Marsland, S. \& Cox, M. P. 2018 Post-marital residence patterns show lineage-specific evolution. Evolution and Human Behavior 39, 594-601. (DOI:10.1016/j.evolhumbehav.2018.06.002).

834 Phil. Trans. R. Soc. B, 20180077. (DOI:https://doi.org/10.1098/rstb.2018.0077).

835 [51] Borgerhoff Mulder, M. 1995 Bridewealth and its correlates: Quantifying changes over time. Current Anthropology 36, 573-603.

[52] Caldwell, J. C., Reddy, P. H. \& Caldwell, P. 1983 The causes of marriage change in South India. Population studies 37, 343-361.

[53] Macfarlan, S. J., Quinlan, R. J. \& Post, E. 2019 Emergent matriliny in a matrifocal, patrilineal population: a male coalitionary perspective. Philosophical Transactions of the Royal Society B: Biological Sciences 374, 20180073. (DOI:doi:10.1098/rstb.2018.0073).

[54] Shenk, M. K., Begley, R. O., Nolin, D. A. \& Swiatek, A. 2019 When does matriliny fail? The frequencies and causes of transitions to and from matriliny estimated from a de novo coding of a cross-cultural sample. Philosophical Transactions of the Royal Society B 374, 20190006.

[55] Collard, M., Shennan, S. J. \& Tehrani, J. J. 2006 Branching versus blending in macroscale cultural evolution: A comparative study. In Mapping our Ancestors: Phylogenetic Approaches in Anthropology and Prehistory (eds. C. P. Lipo, M. J. O'Brien, M. Collard \& S. J. Shennan). New York, Aldine Transaction.

[56] Atkinson, Q. D., Coomber, T., Passmore, S., Greenhill, S. J. \& Kushnick, G. 2016 Cultural and Environmental Predictors of Pre-European Deforestation on Pacific Islands. PLOS ONE. (DOI:http://dx.doi.org/10.1371/journal.pone.0156340).

[57] Pagel, M., Atkinson, Q. D. \& Meade, A. 2007 Frequency of word-use predicts rates of lexical evolution throughout Indo-European history. Nature 449, 717-720.

[58] Griffiths, P. E. \& Neumann-Held, E. M. 1999 The many faces of the gene. Bioscience 49, 656-662.

[59] Turchin, P., Currie, T. E., Whitehouse, H., François, P., Feeney, K., Mullins, D., Hoyer, D., Collins, C., Grohmann, S., Savage, P., et al. 2018 Quantitative historical analysis uncovers a single dimension of complexity that structures global variation in human social organization. PNAS 115, E144-E151. (DOI:10.1073/pnas.1708800115\%J Proceedings of the National Academy of Sciences).

862 R., Ross, R. M., Larson, J. \& Baines, J. 2019 Complex societies precede moralizing gods 863 throughout world history. Nature 568, 226-229. 
864

865

866

867

868

869

870

871

872

873

874

875

876

877

878

879

880

881

882

883

884

885

886

887

888

889

890

891

892

893

894

895

896

897

898

899

900

901

902

903

904

905

906

907

908

909

[61] Miranda, L. \& Freeman, J. 2020 The two types of society: Computationally revealing recurrent social formations and their evolutionary trajectories. PLOS ONE 15, e0232609. (DOI:10.1371/journal.pone.0232609).

[62] Shin, J., Price, M. H., Wolpert, D. H., Shimao, H., Tracey, B. \& Kohler, T. A. 2020 Scale and information-processing thresholds in Holocene social evolution. Nature Communications 11, 2394. (DOI:10.1038/s41467-020-16035-9).

[63] Kappeler, P. M., Clutton-Brock, T., Shultz, S. \& Lukas, D. 2019 Social complexity: patterns, processes, and evolution. (Springer.

[64] Sanderson, M. J., Purvis, A. \& Henze, C. 1998 Phylogenetic supertrees: assembling the trees of life. Trends in Ecology \& Evolution 13, 105-109.

[65] Duda, P. \& Zrzavý, J. 2016 Human population history revealed by a supertree approach. Scientific reports 6, 29890.

[66] Fortunato, L. \& Jordan, F. 2010 Your place or mine? A phylogenetic comparative analysis of marital residence in Indo-European and Austronesian societies. Phil. Trans. R. Soc. B. 365, 39133922. (DOI:doi:10.1098/rstb.2010.00173913).

[67] Ross, C., Strimling, P., Ericksen, K., Lindenfors, P. \& Borgerhoff Mulder, M. 2016 The Origins and Maintenance of Female Genital Modification across Africa. Human Nature 27, 173200. (DOI:DOI 10.1007/s12110-015-9244-5).

[68] Butler, M. A. \& King, A. A. 2004 Phylogenetic Comparative Analysis: A Modeling Approach for Adaptive Evolution. American Naturalist 164, 683-695.

[69] Minocher, R., Duda, P. \& Jaeggi, A. V. J. E. 2018 Explaining marriage patterns in a globally representative sample through socio-ecology and population history: A Bayesian phylogenetic analysis using a new supertree of human cultures. Evolution and Human Behavior.

[70] Boettiger, C., Coop, G. \& Ralph, P. 2012 Is your phylogeny informative? Measuring the power of comparative methods. Evolution: International Journal of Organic Evolution 66, 22402251.

[71] Pagel, M., Meade, A. \& Barker, D. 2004 Bayesian estimation of ancestral character states on phylogenies. Systematic biology 53, 673-684.

[72] Holden, C. J., Meade, A. \& Pagel, M. 2005 Comparison of maximum parsimony and Bayesian Bantu language trees. In The Evolution of Cultural Diversity (eds. R. Mace, C. J. Holden \& S. Shennan), pp. 53-66. London, UCL Press.

[73] Greenhill, S. J., Currie, T. E. \& Gray, R. D. 2009 Does horizontal transmission invalidate cultural phylogenies? . Proceeding of the Royal Society B-Biol. Sci. 276, 2299-2306.

[74] Walker, R. S., Hill, K. R., Flinn, M. V. \& Ellsworth, R. M. 2011 Evolutionary History of $\begin{array}{llll}\text { Hunter-Gatherer } \quad \text { Marriage PlosONE } & \text { Prices. }\end{array}$ (DOI:doi:10.1371/journal.pone.0019066).

[75] Hage, P. \& Marck, J. 2011 Proto-Bantu descent groups. Kinship, Language, and Prehistory. In Kinship, Language, and Prehistory: Per Hage and the Renaissance in Kinship Studies (eds. D. Jones \& M. Bojka), pp. 75-78. Salt Lake City, University of Utah Press.

[76] Puttick, M. N., Ingram, T., Clarke, M. \& Thomas, G. H. 2020 MOTMOT: Models of trait macroevolution on trees (an update). Methods in Ecology and Evolution 11, 464-471.

[77] Cooper, N., Thomas, G. H., Venditti, C., Meade, A. \& Freckleton, R. P. 2016 A cautionary note on the use of Ornstein Uhlenbeck models in macroevolutionary studies. Biological Journal of the Linnean Society 118, 64-77.

[78] Jorgensen, J. G. 1980 Western Indians. Comparative Environments, Languages, and Cultures of 172 Western American Indian Tribes. San Francisco, Freeman. 
910 [79] Bouckaert, R. R., Bowern, C. \& Atkinson, Q. D. 2018 The origin and expansion of Pama911 Nyungan languages across Australia. Nature Ecology \& Evolution 2, 741-749.

912 [80] Ruhlen, M. 1987 A Guide to the World's Languages. 2nd edition ed. CA, Stanford University 913 Press.

914 [81] Mathew, S. \& Perreault, C. 2015 Behavioural variation in 172 small-scale societies indicates 915 that social learning is the main mode of human adaptation. Proceedings of the Royal Society B:

916 Biological Sciences 282, 20150061.

917 [82] Revell, L. J. 2012 phytools: an R package for phylogenetic comparative biology (and other 918 things). Methods in Ecology and Evolution 3, 217-223.

919 [83] Paradis, E. \& Schliep, K. 2019 ape 5.0: an environment for modern phylogenetics and 920 evolutionary analyses in R. Bioinformatics 35, 526-528.

921 [84] Pagel, M. \& Meade, A. 2006 Bayesian analysis of correlated evolution of discrete characters 922 by reversible-jump Markov chain Monte Carlo. American Naturalist 167, 808-825.

923 [85] Beaulieu, J. M. \& O'Meara, B. 2019 OUwie: Analysis of Evolutionary Rates in an OU 924 Framework. R package version 1.57. (DOI:https://CRAN.R-project.org/package=OUwie).

925 [86] Hadfield, J. D. 2010 MCMC Methods for Multi-Response Generalized Linear Mixed Models: 926 The MCMCglmm R Package. Journal of Statistical Software 33, 1-22.

927 [87] Botero, C. A., Gardner, B., Kirby, K. R., Bulbulia, J., Gavin, M. C. \& Gray, R. D. 2014 The 928 ecology of religious beliefs. Proceedings of the National Academy of Sciences 111, 16784-16789. 929 [88] Evans, C. L., Greenhill, S. J., Watts, J., Botero, C. A., Gray, R. D. \& Kirby, K. R. this issue 930 The Uses and Abuses of Tree Thinking in Cultural Evolution. 


\section{S1) WNAI phylogeny}

The figure shows the phylogenetic relationships among the 172 Western North American societies.

The colors reflect whether societies and their ancestors lived in forests (red) or not (black).

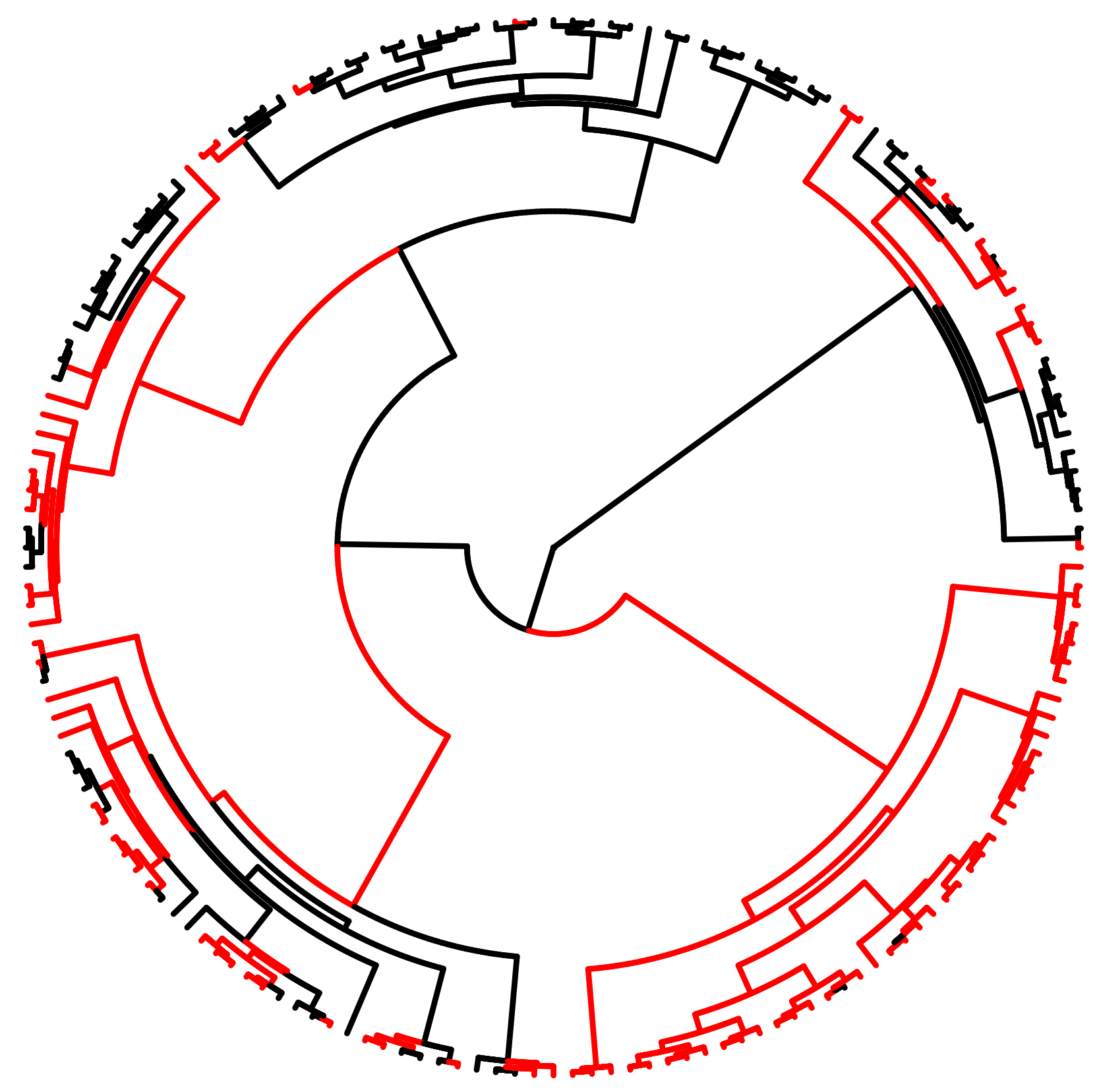




\section{S2) Pama-Nyungan phylogeny}

The figure shows the phylogenetic relationships among the 306 Western Pama-Nyungan societies. The colors reflect whether societies and their ancestors are food-producers (red) or huntergatherers (black).

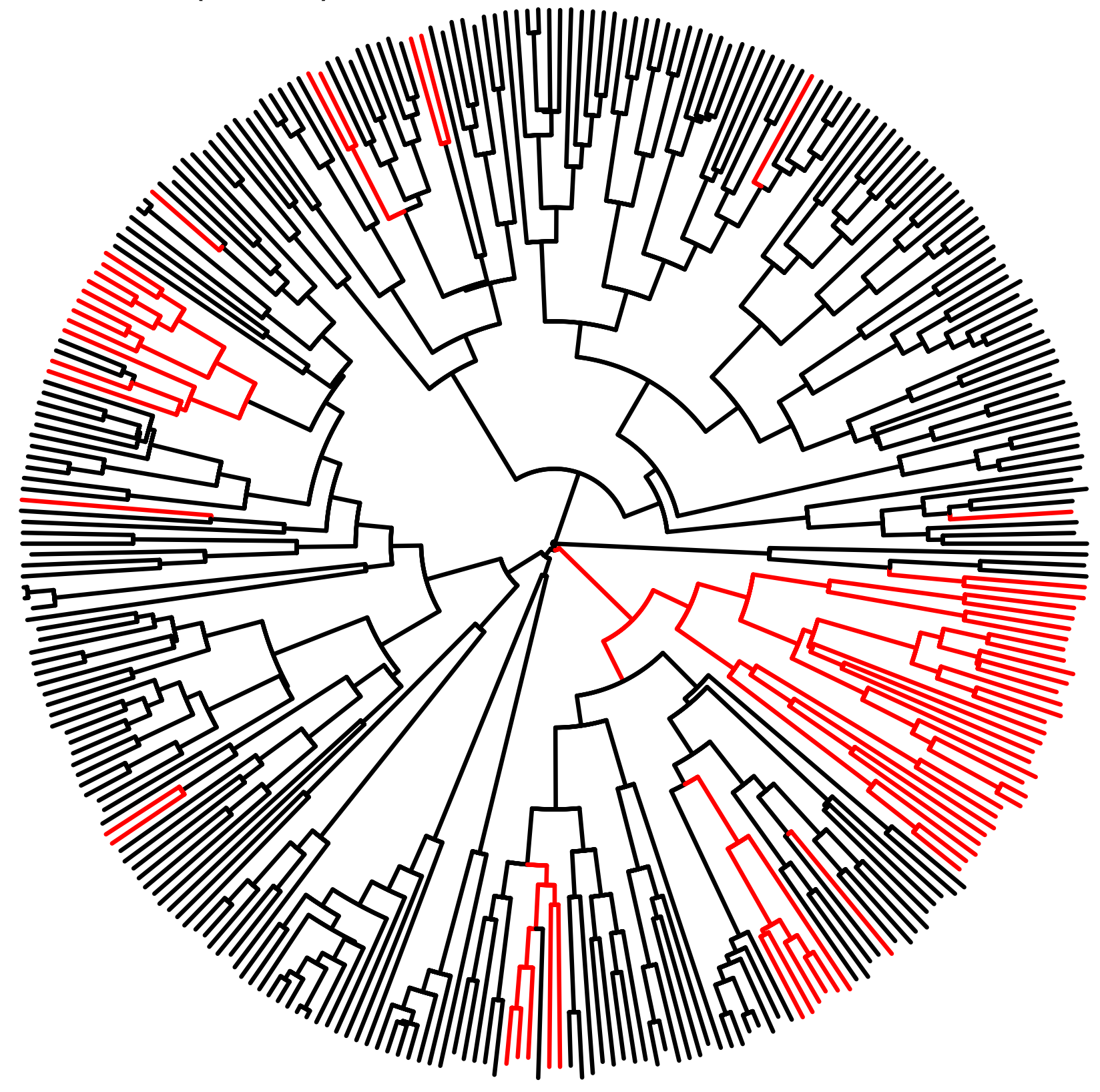




\section{S3) Example of property of the trait}

\section{horizontal transmission within clade}

actual history data available inferred history

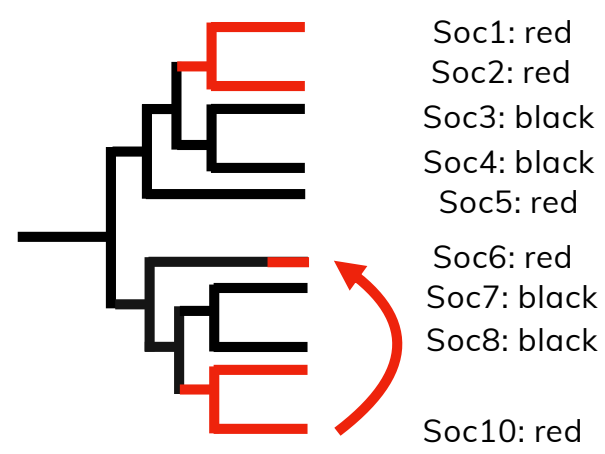

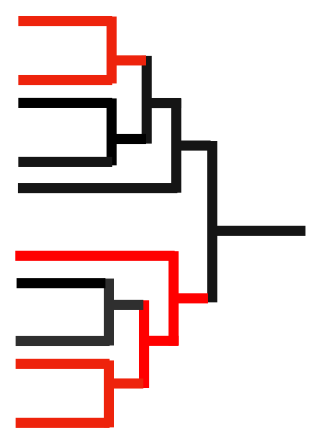

Horizontal transmission of states within clades can homogenize societies within a clade, enhancing differences among societies in different socioecologies and clades.

\section{error rates discrete trait}

direction of change

Type 1: false positive

WNAI PamaNy

$+74 \% \quad+58 \%$

Type 2: false negative

$$
\text { WNAI PamaNy }
$$$$
+36 \%
$$$$
-21 \%
$$

socioecological influences

Type 1: false positive

$$
\begin{array}{ll}
\text { WNAl } & \text { PamaNy } \\
+33 \% & +33 \%
\end{array}
$$

Type 2: false negative

$\begin{array}{ll}\text { WNAl } & \text { PamaNy } \\ +4 \% & +/-0 \%\end{array}$

lineage differences

Type 1: false positive WNAl PamaNy

$+7 \% \quad+9 \%$

Type 2: false negative

WNAI PamaNy

$-13 \%$ $-18 \%$

\section{error rates continuous trait}

\section{direction of change}

Type 1: false positive

WNAI PamaNy $+70 \% \quad+38 \%$

Type 2: false negative $\begin{array}{lc}\text { WNAl } & \text { PamaNy } \\ -41 \% & -26 \%\end{array}$

\section{$\underline{\text { socioecological influences }}$}

Type 1: false positive

$$
\begin{array}{ll}
\text { WNAl } & \text { PamaNy } \\
+/-0 \% & +44 \%
\end{array}
$$

Type 2: false negative

$$
\text { WNAl PamaNy }
$$$$
+99 \%
$$

lineage differences

Type 1: false positive WNAI PamaNy $+57 \% \quad+36 \%$

Type 2: false negative WNAI PamaNy $+/-0 \% \quad+/-0 \%$ 


\section{S4) Example of property of the trait}

\section{horizontal transmission from geographic neighbor}

actual history data available inferred history

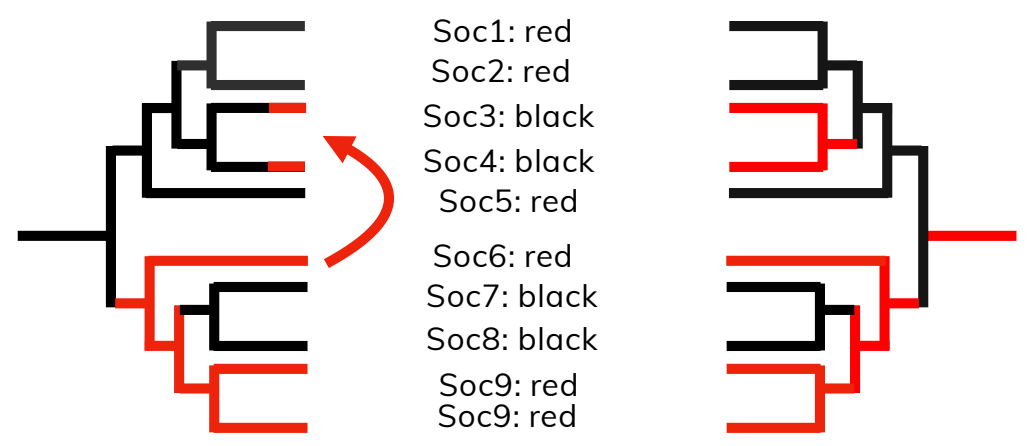

Geographic neighbours are often not the same as phylogenetic neighbours, which can introduce divergent states into clades, biasing the inference of internal nodes.

\section{error rates discrete trait}

\section{direction of change}

Type 1: false positive

$$
\text { WNAl PamaNy }
$$$$
+45 \% \quad+35 \%
$$

Type 2: false negative

$$
\begin{array}{lc}
\text { WNAl } & \text { PamaNy } \\
+19 \% & -21 \%
\end{array}
$$

\section{ecological influences}

Type 1: false positive

$$
\begin{array}{lc}
\text { WNAl } & \text { PamaNy } \\
-10 \% & -18 \%
\end{array}
$$

$-18 \%$

Type 2: false negative

$\begin{array}{cc}\text { WNAl } & \text { PamaNy } \\ +3 \% & +6 \%\end{array}$

lineage differences

Type 1: false positive

WNAl PamaNy

$-10 \% \quad-8 \%$

Type 2: false negative

$$
\text { WNAI }
$$$$
-25 \%
$$

\section{error rates continuous trait}

\section{direction of change}

Type 1: false positive

WNAI PamaNy $+87 \% \quad+98 \%$

Type 2: false negative

$$
\text { WNAI PamaNy }
$$$$
-42 \%
$$

\section{ecological influences}

Type 1: false positive

$$
\begin{array}{cc}
\text { WNAl } & \text { PamaNy } \\
+7 \% & +36 \%
\end{array}
$$

Type 2: false negative

$$
\text { WNAI PamaNy }
$$

$+89 \%$ lineage differences

Type 1: false positive

$$
\begin{array}{ll}
\text { WNAl } & \text { PamaNy } \\
+82 \% & +29 \%
\end{array}
$$

Type 2: false negative

$$
\text { WNAI PamaNy }
$$$$
+/-0 \% \quad+/-0 \%
$$ 


\section{S5) Example of property of the trait fewer variants of the trait}

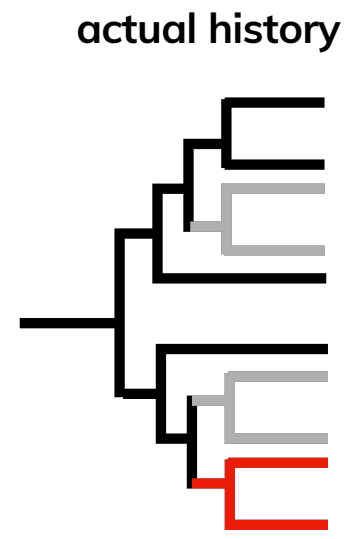

$$
\begin{gathered}
\text { data available } \\
\text { Soc1: black } \\
\text { Soc2: black } \\
\text { Soc3: black } \\
\text { Soc4: black } \\
\text { Soc5: black } \\
\text { Soc6: black } \\
\text { Soc7: black } \\
\text { Soc8: black } \\
\text { Soc9: red } \\
\text { Soc10: red }
\end{gathered}
$$$$
\text { inferred history }
$$

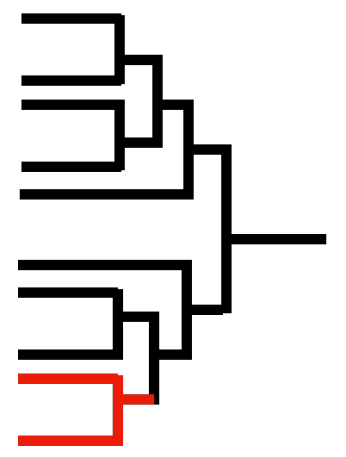

Grouping variants together into single categories (e.g. all shades of grey classified as black) reduces power, potentially suggesting stronger differences among lineages

\section{error rates discrete trait}

direction of change

Type 1: false positive

WNAI PamaNy

$-3 \% \quad-2 \%$

Type 2: false negative ecological influences

Type 1: false positive

WNAI PamaNy

$-1 \% \quad+/-0 \%$

Type 2: false negative

WNAI

$-2 \%$

PamaNy

$+3 \%$ lineage differences

Type 1: false positive

WNAl PamaNy

$-2 \%+10 \%$

Type 2: false negative

WNAI PamaNy

$+6 \%$ 